\title{
Prenatal diagnosis of congenital syphilis ina pregnancy complicated with secondary syphilis in third trimester: A Case Report
}

\author{
Jayawardena GRMUGP ${ }^{1}$, Ranatunga R G J D ${ }^{1}$, Gunarathna SMSG ${ }^{1}$, Wickramasinghe $\mathrm{P}^{2}$ Dias $\mathrm{T}^{1,3}$
}

\section{INTRODUCTION}

Congenital syphilis is still a cause of perinatal morbidity and mortality ${ }^{1}$. Untreated maternal infection leads to adverse pregnancy outcomes, including early fetal loss, stillbirth, prematurity, low birth weight, neonatal and infant death, and congenital disease among newborns. Clinical manifestations of congenital syphilis are influenced by gestational age, stage of maternal syphilis, maternal treatment, and immunological response of the fetus 2, 3. It has been traditionally classified in early congenital syphilis and late congenital syphilis. Diagnosis of maternal infection is based on clinical findings, serological tests, and direct identification of treponemes in clinical specimens. Adequate treatment of maternal infection is effective for preventing maternal transmission to the fetus and for treating fetal infection. Prenatal diagnosis of congenital syphilis includes noninvasive and invasive diagnosis. Serological screening during pregnancy and during preconception period should be performed to reduce the incidence of congenital syphilis.

1. North Colombo Teaching Hospital Ragama

2. District General Hospital Hambanthota

3. Faculty of Medicine, University of Kelaniya

Key words

Congenital syphilis, intrauterine diagnosis of congenital syphilis, intrauterine blood transfusion, secondary syphilis in pregnancy, screening for syphilis in pregnancy

Correspondence: Dr Tiran Dias

E-mail: thiran_dias@yahoo.com

Competing interests: None

\section{CASE REPORT}

A sixteen year old unmarried primi gravida was referred from General Hospital Hambantota for specialized management of hydrops fetalis at 32 weeks of gestation. She was diagnosed as having secondary syphilis with condylomata lata lesions and had a positive VDRL test and treated with one dose of intramuscular benzathine penicillin two days prior to the transfer. Ultrasound scan was suggestive of fetal hydrops and Doppler showed rising middle cerebral artery peak systolic velocity. Fetal anaemia was suspected and cordocentesis has been performed after setup necessities for intrauterine transfusion of packed red cells. Anaemia was confirmed with a haemoglobin of $6.3 \mathrm{~g} / \mathrm{dl}$ and haematocrit of $18.9 \%$ and fetal blood transfusion has been performed successfully. Fetal blood was drawn during the procedure has sent for VDRL and showed a titre of R 64 while mother had a titre of $R 8$, which is a fourfold increase in the VDRL proving the diagnosis of congenital syphilis. Despite maternal antibiotic treatment and intrauterine management of fetal complications intrauterine death occurred at 33 weeks of gestation.

\section{CONCLUSION}

Syphilis infection during pregnancy still represents a worldwide public health problem. It is recommended in international and local guidelines that antenatal syphilis screening should be done at the first visit and again at 32-36 weeks, if the woman is at risk for syphilis ${ }^{4}$. Moreover, any woman who delivers a stillborn infant after 20 weeks' gestation should be tested for syphilis.

The diagnosis of congenital syphilis can be difficult, as maternal nontreponemal and treponemal IgG antibodies can be transferred through the placenta to the fetus, complicating the interpretation of reactive serologic tests for syphilis in neonates. Therefore, treatment decisions frequently must be made on the basis of

1. Identification of syphilis in the mother

2. Adequacy of maternal treatment

3. Presence of clinical, laboratory, or radiographic evidence of syphilis in the neonate

4. Comparison of maternal (at delivery) and neonatal nontreponemal serologic titers using the same test, preferably conducted by the same laboratory. Any neonate at risk of congenital syphilis should receive a full evaluation and testing for HIV infection.

All neonates born to a woman who have reactive serologic tests for syphilis should be examined thoroughly for evidence of congenital syohilis. (eg; non-immune hydrops,jaundice, hepatosolenomegay, rhinitis, skin rash and pseudoparalysis of an extremity) Pathological examination of the placenta or umbilical cord using specific staining(eg silver) or a Treponema pallidum PCR test using a CLIA- validated test should be considered; DFA-TP reagents are not available. Darkfield microscopic examination or PCR testing of suspicious lesions or body fluids (e.g.bullous rash and nasal discharge) also should be performed. In addition to these tests, for still infants, skeletal survey demonstrating typical osseous lesions might aid in the diagnosis of congenital syphilis ${ }^{5}$.

Penicillin is the treatment for pregnancies complicated with syphilis. In pregnancy, the rate of Jarisch-Herxheimer Reaction is the same as in non-pregnant. It is an acute febrile illness resolving in 24 hours. This is common in early syphilis especially in secondary syphilis where spirochetaemia is high. The 
uterine contractions appear to occur secondary to the development of fever. Fetal heart rate decelerations are also reported occurring about $40 \%{ }^{5}$. Preconception serological tests for syphilis could represent the key to reduce the incidence of congenital syphilis. Management of syphilis during pregnancy includes both maternal antibiotic therapy and fetal management of possible complications like anaemia and ascites.
This case clearly tells us the importance of prevention of sexually transmitted infections in pregnancy which is a very comprehensive process starting from education of young males and females on STIs and HIV, preventing infections in young age groups, identification of infections in early pregnancy and prompt treatment of mothers and treatment of the new born or as in this case possible intrauterine interventions.

\section{REFERENCES}

1. SexTransm Infect 2000;76:73$79 ; 10,1136 /$ sti,76.2.73

2. Peadiatrics and child health.2000 NovDec5(8)463-469

3. Sexually transmitted diseases ; King K. Holmes, $4^{\text {th }}$ Edition $1573-1613$

4. CDC Sexually Transmitted Disease Treatment Guideline (2015)

5. Update on management of syphilis in pregnancy. BASHH C E G Statement August 2011 